\title{
Estrategias de permeabilidad en proyectos de gerohabitación
}

Alejandro Pérez-Duarte Fernandez ${ }^{1}$ | Bruno Cruz Petit ${ }^{2}$

Recibido: 23-01-2020 | en su versión final: 05-06-2020

Resumen

\begin{abstract}
Ante la demanda creciente de habitación especializada para las personas adultas mayores resulta imprescindible examinar el proyecto habitacional colectivo a partir de criterios no solo geriátricos sino gerontológicos también. Considerando las fragilidades que este grupo puede presentar (falta de contactos interpersonales y/o estimulación) hay aspectos proyectuales que pueden contribuir positivamente. Partiendo de una reflexión sobre la demolición parcial de la residencia De Drei Hoven de H. Hetzberger, modelo en el sector en los años setenta, se discuten aquí nuevos principios de organización abierta al trazado urbano, que dan lugar a un mayor abanico de posibilidades para la vida multigeneracional. En una revisión de aspectos formales, y programáticos en proyectos sobresalientes en este tipo de edificios se detectan tres estrategias; imán de atracción, corta camino y superposición de programas. Subyace en ellas una tendencia relacionada con la voluntad de hacer permeable la edificación, buscando potencializar el contacto social y la vida activa.
\end{abstract}

Palabras clave: Permeabilidad arquitectónica; espacios multigeneracionales; habitación colectiva; adultos mayores

Citación

Pérez-Duarte, A. \& Cruz, B. (2020). Estrategias de permeabilidad en proyectos de gerohabitación. ACE: Architecture, City and Environment, 15(44), 9198. DOI: http://dx.doi.org/10.5821/ace.15.44.9198

1 Doctor en Teoría e Historia de la Arquitectura, profesor-investigador en el ITESO, México (ORCID: 00000001-5500-9828; WoS Researcher ID: Q-6290-2017; Scopus Author ID: 57217491522) ${ }^{2}$ Doctor en Sociología, miembro del Sistema Nacional de Investigadores de México, Nivel 1, docente-investigador de la Universidad Motolinía del Pedregal (ORCID: 0000-0001-8666-2969) Correos de contacto: apdf230174@live.com, investigacion@ump.mx

\section{Permeability Strategies in the Senior Housing Project}

Abstract

Given the growing demand for specialized housing for the elderly, it is essential to consider the housing project based on criteria not only geriatric but also gerontological. Considering fragilities that older people can present, the lack of interpersonal contacts, and/or stimulation, there are certain project aspects that can contribute positively. Starting with a view on the partial demolition of the De Drei Hoven residence by $\mathrm{H}$. Hetzberger, a model in the 1970s, new organizational principles are discussed opened to the urban layout, and which make a wider range with a multi-generational presence possible. In a review of the project and program aspects, three strategies are detected: attraction magnet, road cutting, and program overlapping. There is a tendency related to the will to make the building permeable, seeking to potentialize social contact and active life.

Keywords: Architectural permeability; multi-generational spaces; housing; senior citizens 


\section{Introducción}

Una aproximación arquitectónica al proyecto de habitación colectiva especializada en para persona adultas mayores o gerohabitación $n^{1}$ no puede realizarse sin tener en cuenta, por una parte, las recomendaciones de la geriatría en cuanto a vivienda físicamente adaptada (OMS, 2018), y, por otra parte, los estudios que nos da la gerontología en general, y la gerontología ambiental en particular sobre este segmento de población. Y si bien existe una abundante investigación, con consensos importantes, en torno a temas arquitectónicos de accesibilidad y adaptabilidad de la vivienda, en la intersección entre la arquitectura y los aspectos psicosociales que trata la gerontología encontramos debates abiertos y propuestas aún por explorar. Ello proviene del carácter cultural y relativo que entraña toda propuesta de estilo de vida. Para Havighusrt (1961), la gerontología no puede deslindarse del objetivo de definir lo que es un envejecimiento exitoso, y debe dar consejos generales que guíen acciones particulares; en este caso, la construcción de espacios idóneos para los que se denomina hoy "personas adultas mayores". Este último término, que viene a sustituir a "ancianos", refleja un cambio cultural que incorpora la propuesta que Havighusrt estaba recomendando desde los años sesenta: el "envejecimiento activo" como modelo de envejecimiento exitoso, para desmarcarse de la teoría de la desvinculación -el retiro gradual de la vida profesional como algo necesario, en Cumming y Henry (1961). En el envejecimiento saludable recomendado por la $\mathrm{OMS}^{2}$, que pudiera matizar la presión prescriptiva del modelo de envejecimiento activo, se señala la necesidad de actuar en múltiples sectores (entre ellos el entorno construido) y de fomentar que las personas mayores sigan siendo una ayuda para sus familias, comunidades y economías, disfrutando el máximo tiempo posible de un grado suficiente de autonomía y capacidades funcionales; e interactuando no solo con adultos mayores sino con otros grupos de edad ${ }^{3}$.

Para Beltrán y Rivas (2013) el envejecimiento activo incluye la intergeneracionalidad como uno de sus principios (sería discutible ver si ello es así en todos los casos). Lo interesante para este trabajo es que la intergeneracionalidad abre un camino para replantearse conceptos centrales de la gerontología (Schwarz, 2012, y Wahl, Weisman, 2003, señalaron la necesidad de renovar la disciplina abriendo nuevos caminos teóricos). Ello nos lleva a considerar incluso sugerencias de la llamada "postgerontología" (Moral, 2017) que defiende no priorizar estrictamente los criterios de edad, puesto que la edad no sólo es cronología. En esta línea de trabajo se pueden realizar análisis integrales de relaciones intergeneracionales en contextos familiares, apoyándose en la psicología social (Antonucci et alt., 2011). Los trabajos de relaciones intergeneracionales, como el de Beltrán y Rivas (2013), abren el panorama de la gerontología de una manera similar a la que pueden hacerlo los estudios sobre viviendas para adultos mayores en entornos intergeneracionales (Agudo, 2015), o sobre espacio público intergeneracional (Peace, 2013). La apertura temática que se comenta ha propiciado el tema de la permeabilidad arquitectónica que aquí se trata.

La perspectiva propuesta es la de una gerohabitación que parece vincularse con las inquietudes de las disciplinas anteriormente referidas y las integra en observaciones eminentemente arquitectónicas ${ }^{4}$. El estudio de la gerohabitación (Sabater, 2009; Pérez-Duarte y Cruz, 2017) se

\footnotetext{
1 Término propuesto por T. Sabater (2001) para la vivienda colectiva especializada con perspectiva gerontológica.

2 Se trata de mantener al máximo el tipo de actividades que personas de la tercera edad realizaban en su mediana edad, siendo éstas sustituidas muy gradualmente por otras en la medida que ya no puedan realizarse, sin perder el vínculo con el mundo social (Havighusrt, 1961). La Organización Mundial de la Salud ha realizado varias conferencias en torno al concepto de envejecimiento activo, definido como "el proceso de optimizar las oportunidades de salud, participación y seguridad para mejorar la calidad de vida de las personas de la tercera edad" (OMS, 2002) y al "envejecimiento saludable", otro término que la OMS (2015) ha escogido para trabajar en este tema en el periodo 2015-2030.

${ }^{3}$ Los modelos ambientales que inspiran dichas recomendaciones son aquellos en los que se promueve un ambiente cuyo nivel de exigencia se sitúe en el límite del máximo nivel de competencia posible en un adulto mayor, que lo estimule sin provocarle estrés ambiental (siguiendo modelo ecológico de Lawton, y Nahemow, 1973); también adaptado a las necesidades y gustos del individuo (modelo de congruencia de Kahana, 1975).

${ }^{4}$ Para Izal y Fernández (1990) dichos modelos enfatizan el papel del ambiente como ambiente percibido o subjetivo frente al ambiente real u objetivo, siendo este último visto sobre todo como una variable de tipo independiente (aunque el mismo
} 
centra metodológicamente en una mirada analítica del proyecto sobre su información técnica gráfica -la lectura de plantas- buscando identificar las estrategias que operan como premisas, regularmente aquellas generadoras de espacios socialmente significativos para personas adultas mayores.

Sabater y Guasch (2020) identifican "descriptores" y "operadores" dentro de una mirada inquisitiva de proyectos dirigida a su posible inclusión en bases de datos de edificios, aunque también se puede abrir a un léxico más amplio - "dispositif architectonique" en Eleb o el "pattern" de Ch. Alexander de la década de los setenta. La idea de operador proyectual es también es afín a la de "estrategias proyectuales" (Moneo, 2004), definidas como "mecanismos, procedimientos, paradigmas y artilugios formales" que aparecen con insistencia en los procesos compositivos. K. Frampton (2015) por otro lado, reconoce "categorías" al hacer análisis comparativos de proyectos equivalentes. Lo que se presenta aquí es un trabajo que identifica patrones en el proyecto habitacional especializado, reconociendo directrices que subyacen como principios de organización, facilitando así posibles contrastaciones futuras con estudios de pos-ocupación. La propuesta es la lectura de una selección de proyectos a partir de la noción de la permeabilidad del espacio; un operador que existe desde las morfologías utilizadas por el estructuralismo holandés, referido también algunas veces como "abertura" por H. Hertzberger (2013), pero que, bajo una perspectiva urbana, del edificio y su entorno inmediato ha sido mayormente explorada como permeabilidad (Henao 2015 y Álvarez, 2018).

\section{De Drie Hoven y su demolición}

La reciente demolición del legendario edificio De Drie Hoven, en Ámsterdam, presentado como paradigma en la década de los setenta, levanta preguntas fundamentales sobre las formas habitacionales adecuadas para personas adultas mayores. Tras su culminación en 1974, el edificio proyectado por Hertzberger se convirtió inmediatamente en un modelo, siendo durante muchas décadas uno de los más admirados y citados (Sutherland, 2013). El planeamiento denotaba una sensibilidad y atención espacial con necesidades particulares de usuarios que, hasta poco antes, durante el Movimiento Moderno, no solían diferenciar (Figura 1).

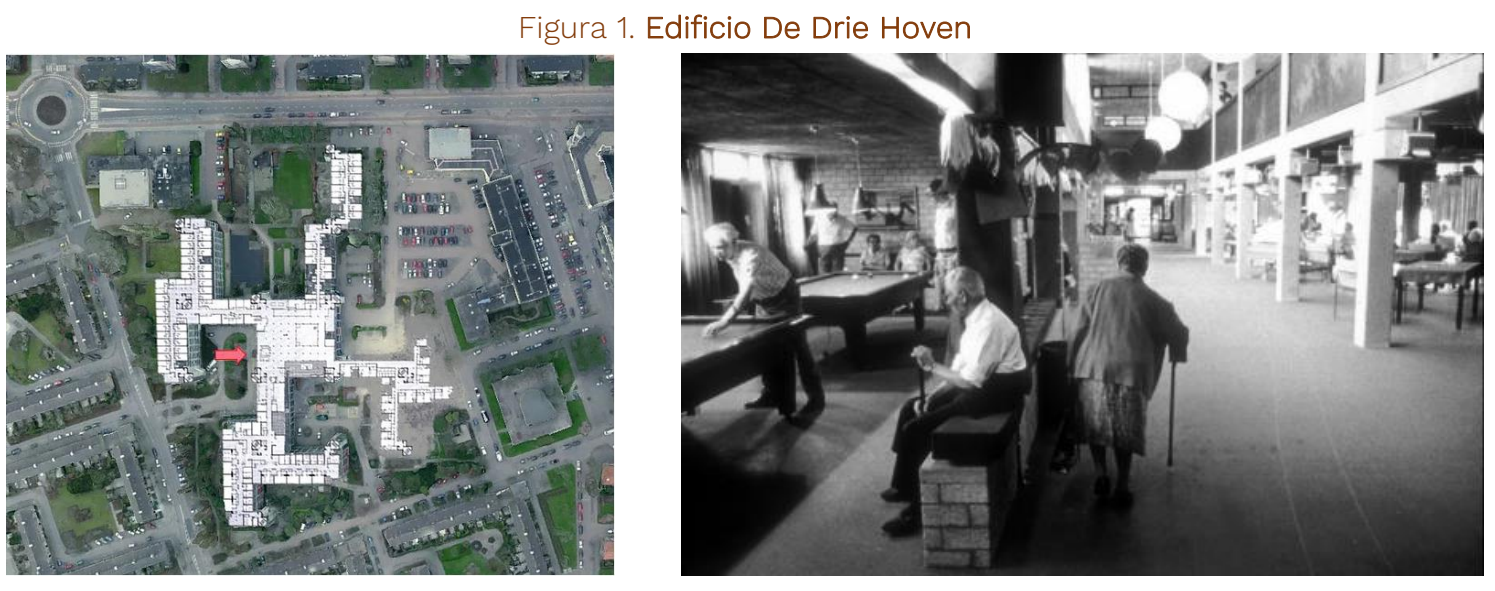

Nota: Cada ala correspondía a una modalidad habitacional para adultos mayores y, en el centro, un lugar de reunión, exclusivo para residentes. Fuente: Campos, Cristina; sobre base GoogleMaps, 2017 / Sutherland, 2013.

Lawton, introdujo la idea de proactividad ambiental para señalar que el entorno puede ser modificado en cualquier momento). Es aquí donde una perspectiva como la gerohabitación, que insiste en los aspectos arquitectónicos del espacio residencial, puede ser útil. 
Desde la misma vista aérea se podían apreciar sus particularidades. La implantación del edificio respondía a una forma de cruz, con cuatro alas, cada una adecuada a una tipología de habitación y a diferentes etapas de la vejez; matrimonios, por un lado, solteros o viudos con autonomía por otro y vida asistida, con mayor apoyo y cuidados en otro. Las habitaciones estaban unidas mediante extensos corredores, generando alas que convergían en un espacio central proyectado para generar intercambios interpersonales. En palabras del arquitecto, era como una "plaza de un pueblo". Los corredores formaban recovecos y recodos, dilatando el espacio como forma de fomento de los encuentros entre residentes. En el De Drei Hoven la estimulación y lucha contra la soledad se resolvían creando una comunidad cerrada de personas en una situación similar, con una amplia área colectiva para actividades improvisadas, de permanencia o convivencia ${ }^{5}$. Del edificio, quizá hoy se diría que respondía a la perfección a la idea del aging-together (Sabater y Maldonado, 2001), procurando evitar el cambio traumático de domicilio. Sin embargo, la opción de la residencia especializada, con ventajas evidentes en cuanto a asistencia médica para fases avanzadas del envejecimiento, ha colisionado con el deseo, regularmente común, de envejecer en el propio domicilio y de manteniendo los lazos significativos sociales del lugar.

El aclamado edificio De Drei Hoven ha desaparecido casi en su totalidad, y algunas de sus alas han liberado espacio para una nueva propuesta de proyecto. La demolición de las torres principales ha sido interpretada tanto en términos de crisis de un modelo tanto arquitectónico como político y económico. En Holanda, al mismo tiempo que se consideró que un programa de atención a las personas mayores tan fuertemente subsidiado era insostenible, se transfirió la responsabilidad desde el nivel regional al municipal (Allen Brooks, 2018). El recorte presupuestal fue paralelo a una apuesta por los servicios de cuidado en el mismo hogar. Muchos centros de cuidado tuvieron que reducir su tamaño o cerrar. Y el enorme De Drei Hoven se ha tenido que adaptar a esta tendencia con bloques de menor tamaño.

Hertzberger, por su parte, opinó disgustado que la reducción del área pública de los ancianos era un indicador del creciente individualismo que refleja el proyecto nuevo (1994, p.4). Se podría argumentar que el área pública que comenta no era exactamente pública, sino semipública, dirigida a un gueto de ancianos. Estudios recientes indican que este tipo de residencias no son interesantes para gran parte de la población de adultos mayores ${ }^{6}$. Si el futuro del De Drie Hoven está llegando a su fin, habrá que repensar el entendimiento espacial de este subgénero habitacional. De hecho, es fácil de prever el surgimiento de "nuevos conceptos de habitación adecuada para la tercera edad" (Brooks, 2018).

\section{De Overloop y la permeabilidad espacial}

El mismo Hertzberger, en una posterior realización, tuvo el acierto de adaptarse a nuevas consideraciones. Su segunda realización en el campo de residencias para adultos mayores, en la década de los ochenta, De Overloop, muestra una solución que contrasta de forma evidente con De Drei Hoven. Esta vez, el proyecto genera una estructura espacial que busca el diálogo con el entorno; usando palabras del propio Hertzberger (2013), se trata de una organización "abierta".

\footnotetext{
${ }^{5}$ Una innovación significativa aparecía en la línea de fachada interna frente al corredor, siendo ésta resuelta con un umbral profundo y la puerta del apartamento de dos hojas, tipo holandesa, pudiéndose abrir la hoja superior y mantener contacto visual con la circulación colectiva. Los umbrales, frente al acceso a las habitaciones, eran verdaderos espacios de convivio. Un tapete, un par de sillas y una pequeña mesa por los mismos residentes creaba el sentido de apropiación, donde dos ancianos podían sentarse ahí y pasar largas horas observando a los demás, pasando frente al corredor; viendo movimiento y vida, como ocurre típicamente en pueblos tradicionales.

${ }^{6}$ Los espacios de convivencia existentes dentro de un modelo de habitación colectiva son un atractivo solo para ciertas personas, como sostiene Vasara (2015), quien argumenta que no deben ser hechas generalizaciones, cuestionando así incluso el principio del aging-together.
} 
Las premisas arquitectónicas operan bajo un principio diferente, pues en lugar de generar un núcleo cerrado y ensimismado, se postula un centro geométrico poroso, una plaza que permite el paso libre y de cruce para cortar camino entre dos puntos de la ciudad (Figura 2). El trazado permite una trayectoria de un lado a otro de la manzana, en una configuración que se integra más al barrio. Si la plaza de De Overloop es el equivalente a la zona de reunión del De Drie Hoven, el tratamiento que recibe es radicalmente diferente.

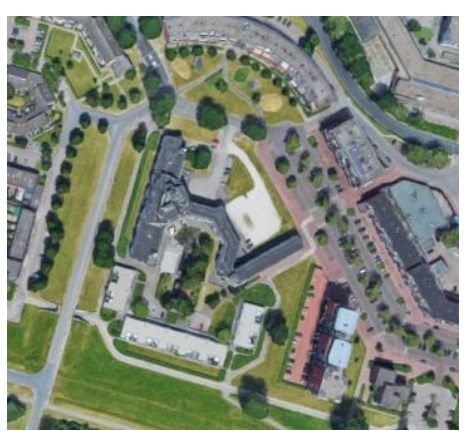

Figura 2. Edificio De Overloop

Nota: De Overloop extiende un ala, como buscando conectarse con la ciudad. Fuente: Google Maps / Cohelo Brito en analisecriticaarquitetura.wordpress.com

Comparando otros aspectos, De Overloop es menos masivo, con menor número de residentes y presenta un criterio binario de las tipologías de habitación, con solo dos formulaciones de células de apartamentos. Por un lado, las que responden a una vida independiente, para aquellos residentes autónomos, con posibilidad de contacto inmediato con el exterior, alrededor de una plaza abierta. Por otro lado, apartamentos organizados a lo largo de un corredor cerrado por ambos lados, con facilidad de cuidados y atención de forma más inmediata. Hay dos zonas para reunión; por un lado, un hall desde donde se prologan los corredores cerrados mencionados; una organización convergente similar a la del De Drie Hoven. Por otro, la plaza exterior, que agrupa las habitaciones de vida independiente. En esta última se puede detectar una secuencia lineal de habitaciones sobre una diagonal, que acompaña al recorrido natural hasta una zona comercial de la ciudad al otro lado de la calle. Se trata de una valiosa conexión que facilita, por ejemplo, ir de compras en un recorrido a pie?.

La comparación entre Drei Hoven y Overloop ilustra un cambio de tendencia en el proyecto habitacional para adultos mayores e introduce en el principio de la permeabilidad, que se propone observar en los siguientes apartados enfatizando conexiones que propician una presencia de grupos etarios diversos.

Pero antes de proceder, es importante considerar la diferencia entre la noción de un espacio multigeneracional e intergeneracional (Thang y Kaplan, 2013). Mientras el primero puede contener grupos de diferentes edades, y puede facilitarse mediante la permeabilidad arquitectónica, en el segundo se consigue imprimir un sentido espacial al lugar, pudiendo generar un significado en la vida de la persona mediante el intercambio interpersonal. Configuración espacial y programa arquitectónico pueden reunir grupos etarios diferentes, pero la interacción entre ellos necesita un trabajo multidisciplinar integrado con arquitectura.

\footnotetext{
${ }^{7}$ La ciudad donde se ubica De Overloop, es Almere, un municipio consciente de la importancia del espacio público amigable con las personas mayores. Allí opera el organismo Gezondheid Fabriek, dedicado al proyecto TITTAN (Translation, Innovation, \& Technology Transfer in Ageing Network), red apoyada por fondos europeos que tiene como objetivo establecer una red de intercambio de buenas prácticas relativas al envejecimiento activo y saludable entre siete regiones de Europa. Alemere está además próxima a Ámsterdam, una de las ciudades que pertenece a la red promovida por la OMS de Ciudades Adaptadas a las Personas Mayores. Ámsterdam contempla la participación de estas personas en las decisiones urbanísticas, y tiene programas multidisciplinarios que buscan crear una ciudad saludable, combinando criterios de planificación espacial y con los de salud pública.
} 


\section{Imán de atracción al corazón del proyecto}

Otro caso afamado es el edificio Humanitas-Bergweg (Rotterdam, 1993-96, EGM Architecten), desarrollado bajo una clara estrategia de dialogo con su entorno urbano; siendo reconocido como un ejemplo de referencia en el sector, y que para este fin ayuda además a plantear una estrategia de la permeabilidad y que se propone aquí bajo el nombre de imán de atracción, de forma similar a como se ha sugerido en otros trabajos sobre espacios públicos intergeneracionales (Azevedo, 2020). En el Humanitas-Bergweg, al nivel de la acera, el basamento aloja un supermercado e integra en la parte posterior servicios públicos de diferente tipo, como un centro de día para inmigrantes, fisioterapia y educación infantil (Figura 3).

La oferta de servicios alcanza al barrio entero. Pero lo que se desplanta en el primer nivel, sobre el basamento, son dos bloques laminares con 280 unidades de apartamentos en forma de ángulo agudo, generando el espacio que da sentido a todo el proyecto: un gran patio de uso colectivo a cubierto, pero cuyo uso no está restringido a residentes.

Figura. 3. Edificio Humanitas-Bergweg

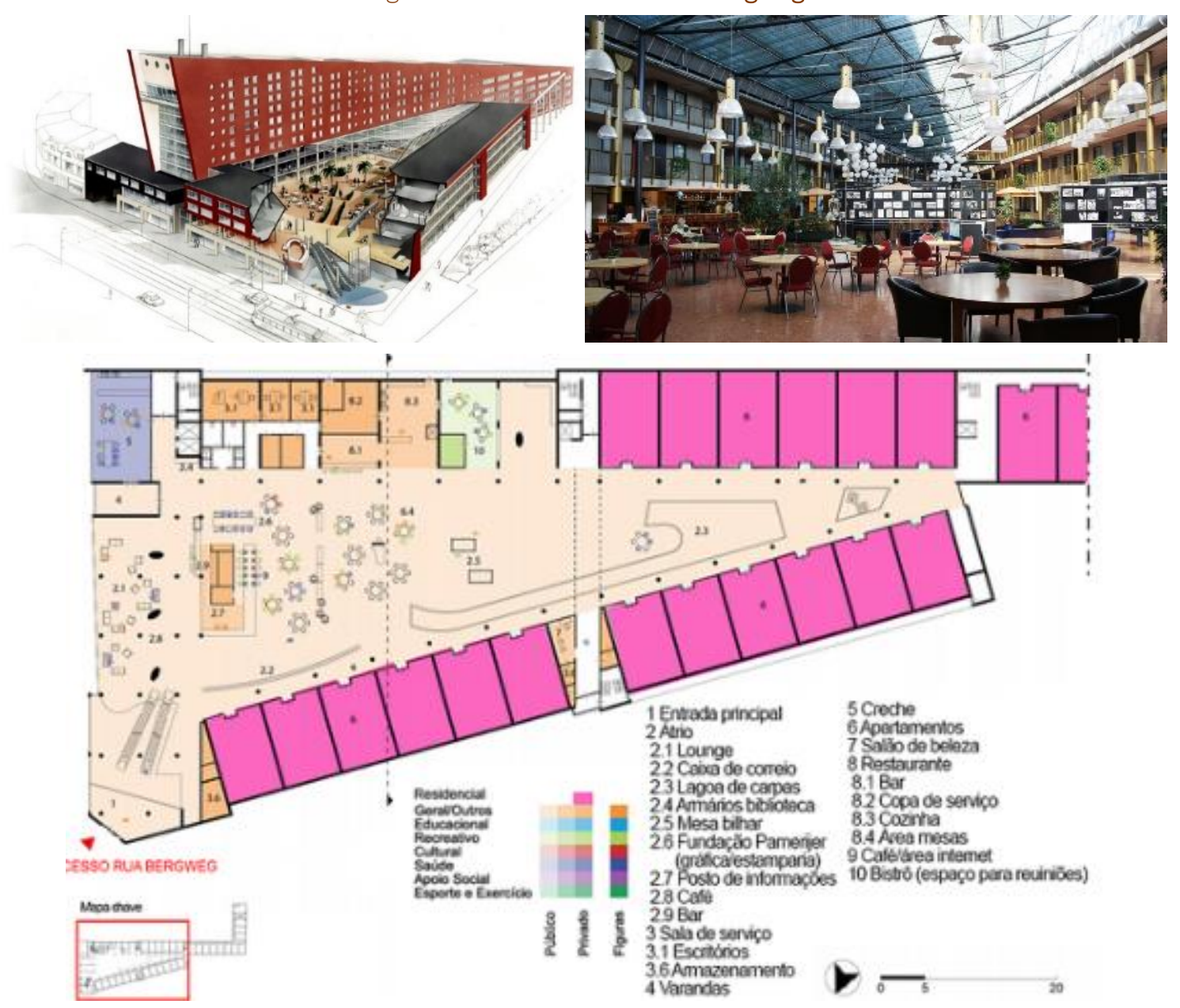

Nota: La esquina del edificio detenta una doble escalera mecánica que conduce a este patio, a cubierto y climatizado, donde se ofrece una gran diversidad de servicios, principalmente de comida y bebidas, generando un potente imán de atracción de residentes del entorno. Fuente: Salvador, en Pérez-Duarte, 2017 (basado en Russen, 2015) 
Dicho patio en forma de triángulo constituye un ámbito intermedio de conexión entre la zona privada de los apartamentos y el exterior; manteniendo un tratamiento que además consigue interiorizar al espacio público de la calle. Por un lado, hay un atractivo en el patio por la oferta de pequeños comercios, restaurantes y servicios, completados además por zonas de permanencia, con lugares para sentarse, café internet y hasta mesas de billar. Por otro lado, hay una facilidad de acceso al público externo mediante dos escaleras mecánicas que conectan directamente con la calle. El resultado en el patio es un espacio con posibilidad de concentrar vitalidad significativa y funciona como un área de condensación social del barrio donde pueden acudir tanto residentes en el edificio como personas externas atraídas por un programa de servicios. Hay una presencia multigeneracional por una facilidad de acceso junto con una buena captación e incitación a la permanencia.

H. Becker (2014), gestor durante muchos años de este edificio, se refiere a parte de la oferta de servicios como el "happy meals" del modelo de "restaurante para ciudadanos de tercera edad". Según explica, el consumo de alimentos, el compartirlos con otras personas, forma parte del rito diario de los usuarios y es capaz no solo de mejorar la calidad de vida sino de crear momentos con significado en el cotidiano. El patio contiene también oferta de alimentos "con atmosferas diferentes" y se ofrecen hasta "25 selecciones diferentes de menús preparados por cocineros de la industria, instruidos para enfocarse en la cualidad y no en la economía apenas” (Becker, 2014, p. 97).

El consumo de cerveza y de vino no solo son ofrecidos, sino promovidos y existe incluso un área para fumar. Becker explica que se debe evitar lo que llama de "dictadura de la dieta y de la salud". El resultado es que, según registros, aproximadamente 40\% de los comensales no suelen ser residentes (Birkbeck, 2014). Por otro lado, cabe apuntar la naturaleza sociópeta del espacio que alienta el contacto (Osmond en Hall, 1966) pues la oferta de pequeños comercios -salón de belleza, correo, papelería, y hasta una guardería- vienen a completar y reforzar, de forma sinérgica, la vitalidad del lugar.

Pero la estrategia se extiende más allá de la solución espacial, pues según Becker, adultos mayores necesitan cosas para discutir, como un evento, una fiesta, una exposición. En 2002, por ejemplo, se representó un musical con título "Mi ciudad vital" organizado e interpretado por clientes, así como por empleados, voluntarios y residentes (Russen, 2015). Otro registro describe un sorprendente evento en el que animales de un zoológico visitaban el patio. El evento, según los cuidadores del Humanitas, tuvo efectos benéficos en los residentes adultos mayores hasta una semana después, con una disminución del número de demandas de cuidados (Birkbeck, 2014).

Más allá la incidencia en la calidad de vida de los residentes, la configuración espacial, política y programa del edificio del Humanitas-Bergweg ha dado impactos económicos positivos: la institución mantiene bajos índices de servicio, con una persona de servicio por cada tres residentes, frente una media de uno a uno de una gestión asistida tradicional holandesa (Birkbeck, 2014).

El convivio en el patio es resultado de un trabajo en diferentes niveles; el edificio facilita y permite la presencia de diferentes grupos etarios y el programa de actividades viene a completarlo para que haya interacción entre éstos. Humanitas-Bergweg se ha convertido en un lugar con significado para algunos residentes, y disfrutan de la vivacidad y de la fuerte dinámica social existente.

Por otro lado, cabe mencionar el edificio mantiene no solo permeabilidad con el exterior, sino que desarrolla una estrategia similar que alcanza las zonas privadas de los pisos superiores, en donde corredores colectivos y apartamentos se abren de forma discreta; un muro poroso puntualmente. Así, por ejemplo, una ventana de la cocina al corredor de circulación colectiva es una forma que incitar intercambios de saludos; lo que Becker refiere como "talking points" que ayudan a integrar a los residentes en una "familia extendida" -uno de los valores que la institución identifica como básicos (Russen, 2015). 


\section{Estrategias multi-generacionales de corta camino y superposición de programa}

La configuración parece invitar al público ajeno del barrio a visitar la plaza ajardinada, en una integración urbana. Dicha pasarela se encuentra además bajo cubierta y cruza de lado a lados el complejo arquitectónico, funcionando como una trayectoria que corta camino de un lado a otro de la manzana (Figura 4).

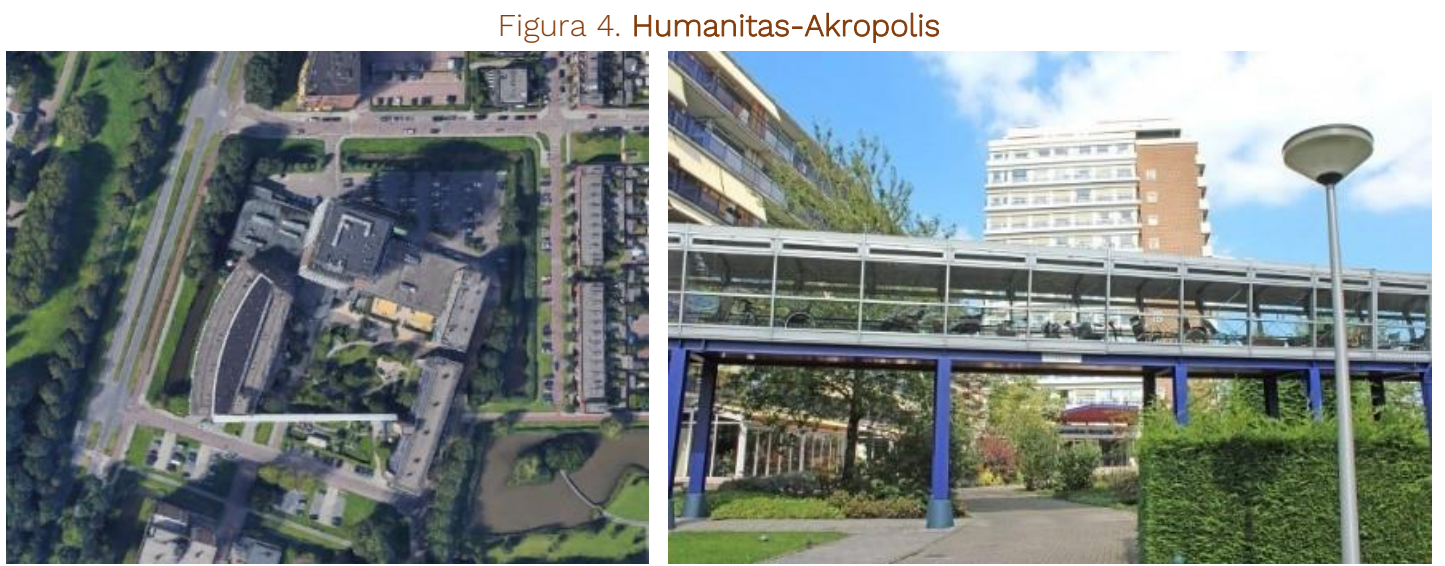

Nota: Humanitas-Akropolis inserta una pasarela a cubierto que corta de lado a lado de la manzana, integrándose con el tejido urbano y haciendo pasar a visitantes por el corazón del conjunto. Fuente: Google Maps

A propósito de la permeabilidad mediante la estrategia de corta camino, una realización más reciente muestra una disposición similar en el centro de residencia para adultos mayores ubicado en Huningue, Francia, de Dominique Coulon \& Associés (2018), detenta un núcleo cruzado por una trayectoria que conecta la calle, de un lado, con el río al otro lado del edificio. Se invita a visitantes a pasar por el centro del edificio, donde el patio bajo cubierta está rodeado de espacios para talleres recreativos. La explicación de los propios arquitectos verbaliza claramente la intención de alcanzar permeabilidad proyectual, explicando una "transparencia a través del edificio" mediante un diagrama analítico (Figura 5).

Figura 5. Complejo de habitación para adultos mayores em Huningue
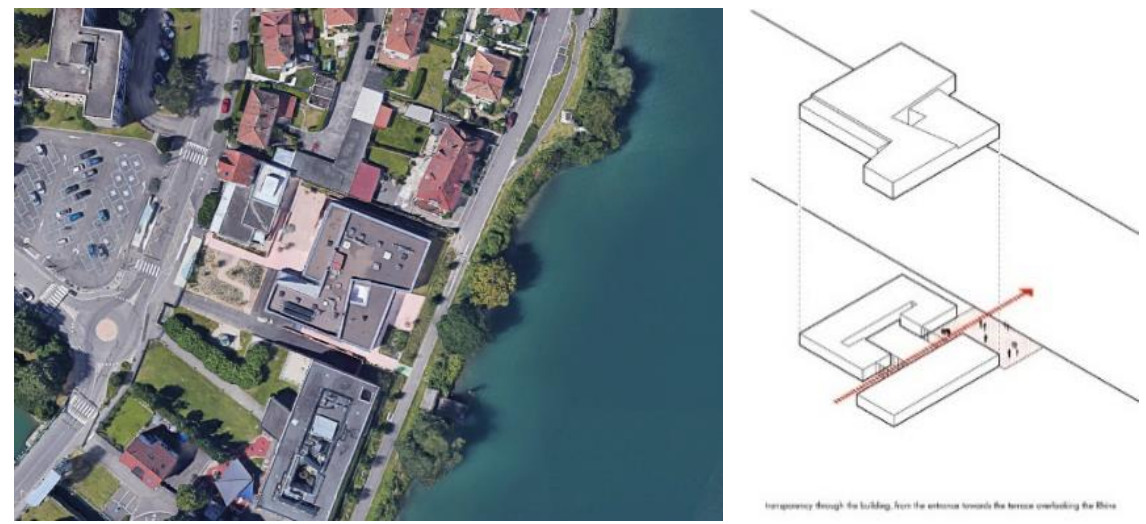

Nota: Estrategia de corta camino que pasa por el corazón del edificio, abriendo una trayectoria de un lado de la calle al río. Fuente: Google Maps / archdaily.com.br

Otra estrategia para incitar presencia multigeneracional, es la superposición programática de usos, como se puede ver en la selección de servicios del Humanitas-Bergweg. 
Un programa arquitectónico mixto es, en sí mismo, un recurso, lo cual se puede observar también en otro caso más reciente (2013), el edificio De Bouwmeester, en Utrecht, de LEVS Architecten. El proyecto aloja usos tan diversos como jardín de niños, centro de atención psicológica, centro de geriátrica de día y vivienda para adultos mayores, alojando vivienda social en los niveles superiores.

El patio que organiza el proyecto, no obstante, se abre selectivamente, pues solo hay acceso, o pasando a través de la guardería para niños, o desde el centro de día para adultos mayores. Según sus arquitectos "todas las funciones se unen, creando lugares de encuentro y relaciones (visuales). Tanto los jóvenes como los mayores permanecen protegidos y en medio de la sociedad" (Levs, 2013, pág. 4).

Figura 6. Edificio De Bouwmeester (2013, Utrecht), LEVS Architecten
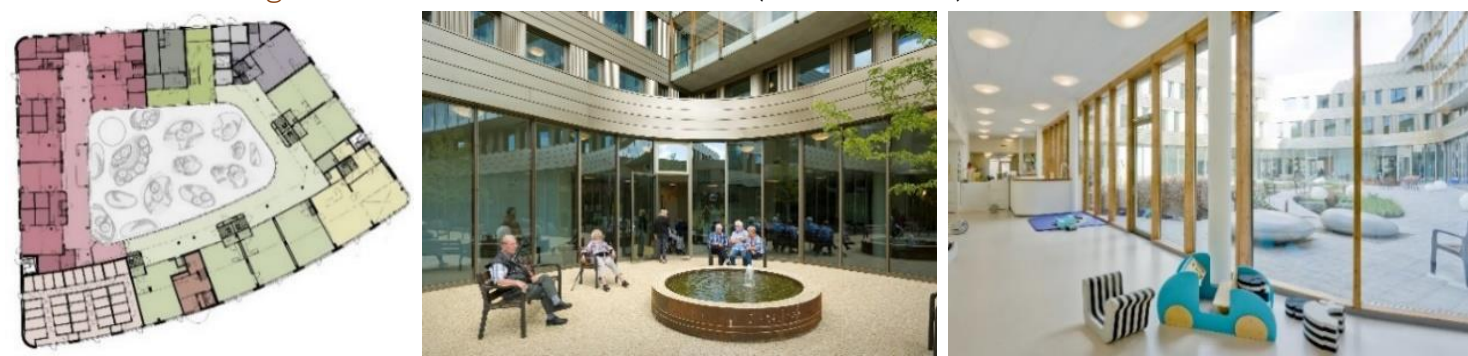

Nota: La planta baja acoge dos usos, jardín de niños (rojo) y centro de día para adulto mayor (verde). Grandes ventanales generan en el patio una conexión visual. Fuente: architizer.com / der Burg

\section{Conclusiones}

En la observación de los casos de estudio se han detectado distintas modalidades y grados de permeabilidad. En términos conceptuales podrían ser descritos como: a) imán de atracción de la comunidad del entorno; b) territorio corta camino; y c) superposición de programas de usos diversos en un mismo espacio.

Figura 7. Representación diagramática de tres soluciones que responden al principio de la permeabilidad con el exterior

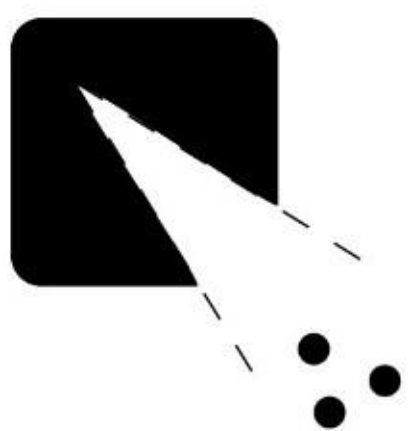

iman de atracción

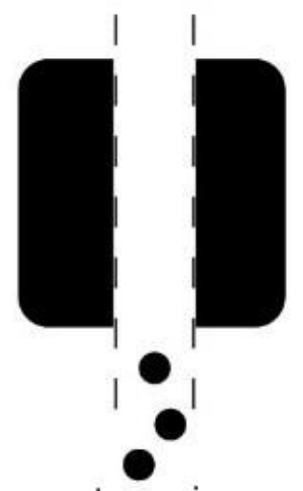

corta-camino

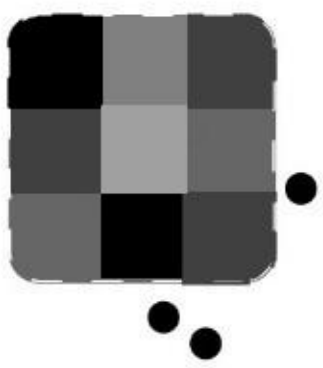

superposición programatica

Fuente: elaboración propia.

Se trata de diferentes soluciones que responden a una misma estrategia proyectual y que operan de forma similar, en el sentido de formar dispositivos que generan contactos entre residentes y visitantes, incentivando y propiciando algunas veces conversaciones fortuitas o contactos puntuales 
-miradas e interacciones breves que son también son una forma de contacto social. Se ha visto en el plano proyectual una suerte de "desmaterialización" de los muros que protegen el interior privado; un deseo para no generar una línea dura entre lo privado y lo público, sino de mezclar estatutos territoriales, dando lugar a zonas semiprivadas o semipúblicas. No hay en los proyectos exactamente un interior versus exterior, sino una zona difusa y ambigua, que funciona como verdaderos mecanismos de congregación social.

La permeabilidad se beneficia de las sinergias positivas con una organización urbana, accesible, incluyente, amigable con los adultos mayores. Se trata de un proyecto que abre espacios en la ciudad en lugar de cerrarlos, a una ciudad que recuerda también a la ciudad abierta de R. Sennett (2007), quien criticó la morfología urbana vertical "moderna" en la que las personas viven y trabajan aisladas en bloques. Sennett apuesta por la membrana porosa, en oposición al muro; incluso pone como ejemplo de "disonancia" aparente pero enriquecedora la plaza de un hospicio para enfermos de SIDA en medio de una calle comercial, lo que hace pensar que estaría a favor de un trazado permeable como el del De Oveloop. La ciudad abierta y la porosidad combaten la zonificación monofuncional (Rossini, F., Roca; E., y Harris, S., 2018). y, si es bien planificada, puede dar lugar a una riqueza de vida, con múltiples actividades en espacios complejos, interesantes a la hora de favorecer espacialmente los propósitos del envejecimiento saludable.

Uno de los recursos de la permeabilidad arquitectónica es fusionar las plantas bajas de las edificaciones con el espacio público colindante al edificio (Henao, 2015; Solà-Morales, 2008) y permitir la continuidad del desplazamiento en el ámbito desde lo público a un espacio con rasgos de semipúblico. De Overloop, así como el caso de Humanitas-Akropolis y el caso de Huningue, optan por otra solución al abrirse hacia su entorno y la ciudad, incorporando el espacio urbano en su corazón. Los efectos son similares, pues se avanza hacia la mejora de las ciudades actuales convirtiéndolas en sistemas abiertos.

No obstante, hay que considerar que en ciudades con espacios públicos más conflictivos las estrategias no pueden ser las mismas. Sería equívoco pensar que las soluciones encontradas puedan ser implantadas de forma idéntica en todo contexto, para cada caso, la permeabilidad con el exterior como principio proyectual de la gerohabitación tendrá que adquirir soluciones específicas en cada caso.

Estudios de gerontología ambiental apuntan por otro lado que, particularmente cuando se trata de espacios residenciales, es importante crear lugares capaces de generar significado en la vida de sus usuarios. Es especialmente en el caso de adultos mayores donde el domicilio debe ser objeto de identificación personal, con fuertes lazos y vínculos con la memoria personal. El caso de Humanitas-Bergweg, por ejemplo, hay un pequeño museo que recrea ambientes domésticos de antaño; una forma de aproximación a la memoria de sus residentes. Un espacio anónimo doméstico, impersonal, sin el sentido de lugar-como lo sería un hospital-impacta negativamente, y el efecto se agrava en un momento de fragilidad del adulto mayor. Así, por ejemplo, considerando los efectos traumáticos del proceso de casser maison -en el que el anciano se ve obligado forzosamente a deshacerse de posesiones adquiridas de su vida para internarse en una habitación menor-, la capacidad de un espacio de generar significado y puede llegar a minimizar sus efectos negativos.

Pasando el umbral de la vivienda, en el ámbito público, hay más riqueza social y capacidad de generación de significados cuando se comparte con otras personas y de diferentes grupos sociales. Un espacio semipúblico o colectivo, con diversidad etaria, muestra mayores posibilidades de intercambio social. En este sentido, la solución del gueto, como el de la configuración del De Drie Hoven, limita y empobrece. 
Aunque el De Drie Hoven ha sido casi completamente demolido, existe aún practicas semejantes al gueto muy extendidas. Tal es el modelo americano de las CCRC y las retirement villages donde, en palabras de un residente, la segregación racial ha venido a ser sustituida por una "segregación etaria" (Thang y Kaplan, 2013) ${ }^{8}$.

Será interesante en futuras investigaciones abordar análisis de proyectos que proponen significados intergeneracionales en configuraciones de integración etaria acompañados de actividades estratégicas. El intercambio social no sucede solamente con la presencia simultánea de los grupos en un mismo lugar, sino que se necesita incitarla y potencializarla. Los casos de estudio aquí anotados posibilitan la presencia multigeneracional, pero una interacción efectiva entre estos no está garantizada, siendo necesaria una perspectiva amplia de trabajo, la cual va más allá de la configuración y programa de la arquitectura.

\section{Autoría}

El primer autor fue responsable por la base de datos informatizada "Gerohabitación" desarrollada bajo el Laboratorio de Movilidad Reducida y Tercera Edad (ITESO/FUMEC). El presente artículo se basa en dicho trabajo. El segundo autor ha enriquecido la mirada en los casos de estudio aquí citados bajo una perspectiva sociológica.

Conflicto de intereses: Los autores declaran que no hay conflicto de intereses.

\section{Bibliografía}

Agudo, M. (2015). Arquitectura intergeneracional. En: Gómez y Méndez, J. M- [et al.] (coord.) Derechos humanos emergentes y periodismo. Sevilla: Equipo de Investigación de Análisis y Técnica de la Información, Universidad de Sevilla, pág. 12-22. Recuperado de: http://hdl.handle.net/11441/36874

Antonucci, T.C. Birditt, K.S.; Sherman, C.W., y Trinh, S. (2011). Stability and change in the intergenerational family: a convoy approach. Ageing and Society, 31(7), 1084-1106. DOI: https://doi.org/10.1017/S0144686X1000098XPu

Azevedo, C. (2020). Urban Public Parks, Magnets for Social and Engagement Across Generations. En Kaplan, M.; Leng T.; Sanchez M., y Hoffman, J. (Eds.). Intergenerational Contact Zones: Place-based Strategies for Promoting Social Inclusion and Belonging. Nueva York: Routledge

Becker, H. (2014). A Taste of Good Living: The Senior Citizens' Restaurant. Delft, Países Bajos: Eburon Publishers.

Beltrán, A. y RIVAS, A. (2013). Intergeneracionalidad y multigeneralidad en el envejecimiento y la vejez. Tabula Rasa. (18), 277-294.

\footnotetext{
8 "No se puede apenas colocarlos [niños y adultos mayores] en un cuarto y pensar que un programa intergeneracional va a comenzar a funcionar, eso requeriría tremendo esfuerzo y tiempo, y también requiere un grupo espacial entrenado [staff] que tenga la voluntad de hacer el esfuerzo" (Hayes, 2003, p. 125 cit. en Thang y Kaplan, 2013).
} 
Birkbeck, D. (2014, mar.). Happy Meals. En: Farrely, L. (ed.), Designing for the Third Age. AD Architectural Design

Brooks, A. (2018, Nov.). Travelling Fellowship. Case Study: De Drie Hoven in "Housing Our Mature Elders". Recuperado de: https://housingourmatureelders.wordpress.com/2018/11/09/case-study-dedrie-hoven/

Cumming, E. Y Henry, E. (1961). Growing old. Nueva York: Basic Books.

Frampton, K. (2015). A Genealogy od Modern Architecture. Zurich: Lars Muller Publishers.

Hall, E. T. (1966). The hidden Dimension. Nueva York, Anchor Books.

Havighurst, H. J. (1961). Successful Aging. The Gerontologist, 1, pág. 8-13.

Hayes, Ch. L. (2003). An Observational Study in Developing an Intergenerational Shared Site Program. Challenges and Insights. Journal of Intergenerational Relationships, 113-132. DOI: https://doi.org/10.1300/J194v01n01 10

Henao Quintero, L. A. (2015, jun.). La permeabilidad de las formas arquitectónicas: los Mercados Municipales de Ciutat Vella y del Eixample de Barcelona. Actas del Seminario Internacional de Investigación en Urbanismo. "VII Seminario Internacional de Investigación en Urbanismo, BarcelonaMontevideo".

Hertzberger, H. (2014). Open versus Closed Structures. En S. Frausto et al: Open Structures: An Introductory Dossier to Dutch Structuralism. Delft University of Technology-Berlage Center for Advanced Studies in Architecture and Design.

Izal, M.; y Fernández., R. (1990). Modelos ambientales sobre la vejez. Anales de Psicología. 6 (2), 181198

Kahana, E. (1975). A Congruence Model of Person Environment Interaction. En P.G. Windley, T. Byerts and E.G. Ernst (Eds.): Theoretical Development in Environments and Aging. Washington, D.C. Gerontological Society.

Lawton, M.P., y Nahemow L. (1973). Ecology and the Aging Process. En C. Eisdorfer y M.P. Lawton. (Eds.): The Psychology of adult development and aging. American Psychological Association, Washington, DC.

Levs Architecten (2013). De Bouwmeester- Utrecht. Recuperado de https://www.levs.nl/projecten/de-bouwmeester-1.html

Moneo, R. (2004). Inquietud Teórica y estrategia proyectual en la obra de ocho arquitectos contemporáneos. Barcelona: Actar.

Pérez-Duarte, A., y Cruz, B. (2017). Gerohabitación, Cohabitación, Indeterminación: Tres Estrategias De Proyecto Para La Tercera Edad / Senior Housing, Cohabitation, Indetermination: Three Project Strategies For The Elderly. Proyecto, Progreso, Arquitectura, (16), 74-87. DOI: https://doi.org/10.12795/ppa2017.i16.05 
Rossini, F.; Roca; E., y Harris, S. (2018). La noción de suelo: una definición de Permeabilidad Urbana en Hong Kong y Barcelona. ACE: Architecture, City and Environment, 13(38), 211-234. DOI: https://doi.org/10.5821/ace.13.38.5432

Rowles, G. D., \& Bernard, M. (Eds.). (2013). Environmental gerontology: Making meaningful places in old age. Springer Publishing Co.

Russen G., H. M. Van (2015). Humanitas Bergweg: een woonzorgcomplex als centrum voor de buurt. [History Thesis]. Holanda: TU Delft. Recuperado de https://www.veldacademie.nl/img/Document/b1/ 72/b1720345-220d-4361-8048-b07498f730be/20150128 Scriptie HumanitasBergweg FINAL.pdf

Sabater, T. y Maldonado, J. (2009). Guía de estudio para la Gerohabitación, la cohabitación y la emancipación, (encargo del Servei de Promoció Económica de la Diputació de Barcelona, DIBA, 1999-2002), Barcelona: Ed. Oficina Multimèdia i publicacions de la ETSAV/ Elisava.

Sennett, R. (2007). La Ciudad Abierta. Otra parte (Argentina), 11, 26-32.

Solà-Morales, M. (2008). De Cosas Urbanas. Barcelona: Gustavo Gili

Sutherland, L. (22 abril 2013) "February 1976: Hertzberger's Framework for Care". The Architectural Review. Recuperado de https://www.architectural-review.com/essays/february-1976-hertzbergersframework-for-care/8646676.article

Vasara, P., (2015). "Not ageing in place: negotiating meanings of residency in age-related housing", Journal of Aging Studies. 25.2 Recuperado de https://www.sciencedirect.com/science/article/abs/pii/S0890406515300116

World Health Organization. (2018). Housing and Health Guidelines. Recuperado de https://apps.who.int/iris/bitstream/handle/10665/276001/9789241550376-eng.pdf 